\title{
Endodualisable and endoprimal finite double Stone algebras
}

\author{
Miroslav Haviar and Hilary A. Priestley \\ Dedicated to the 70th birthday of Tibor Katriñák and to the memory of Rod Beazer
}

Abstract. The finite endodualisable double Stone algebras are characterised, and every finite endoprimal double Stone algebra is shown to be endodualisable.

\section{Introduction}

Double Stone algebras have been extensively studied, both as algebras of interest in their own right and to illuminate studies of properties in universal algebra. The papers [2] [3] 6], 7] by R. Beazer, [23], 24] by I. Düntsch and [27]-29] by T. Katriňák provide a sample of the literature. Further papers on the topic by R. Beazer, to whose memory we dedicate this paper, are included in the bibliography. In the present paper we investigate the related concepts of endoprimality and endodualisability within the variety $\mathcal{D} \mathfrak{S}$ of double Stone algebras. The natural duality theory that we require is summarised in Section 3 . We have deliberately kept this background to a minimum. For more information, and historical references, we refer the reader to the comprehensive monograph 9 by D.M. Clark and B.A. Davey and also to [26].

Let $\mathbf{M}=(M ; F)$ be any algebra. We say that a $k$-ary function $f: M^{k} \rightarrow M$ is End $\mathbf{M}$-preserving if it preserves the action of the endomorphism monoid End $\mathbf{M}$, that is, for every endomorphism $e \in \operatorname{End} \mathbf{M}$,

$$
e\left(f\left(a_{1}, \ldots, a_{k}\right)\right)=f\left(e\left(a_{1}\right), \ldots, e\left(a_{k}\right)\right), \text { for all } a_{1}, \ldots, a_{k} \in M .
$$

The algebra $\mathbf{M}$ is called $k$-endoprimal $(k \geqslant 1)$ if every $k$-ary End $\mathbf{M}$-preserving function on $\mathbf{M}$ is a term function of $\mathbf{M}$. It is clear that each $k$-endoprimal algebra is also $m$-endoprimal for $1 \leqslant m \leqslant k$. Algebras which are $k$-endoprimal for every $k \geqslant 1$ are called endoprimal. Any primal algebra is endoprimal and so, more generally, is any quasiprimal algebra having no proper non-trivial subalgebras. Further, a finite algebra $\mathbf{M}$ is endoprimal if it is endodualisable, that is, if End $\mathbf{M}$ yields a duality

2000 Mathematics Subject Classification: Primary: 08A35, 08A40, 06D15; Secondary: 08 C05.

Key words and phrases: Double Stone algebra, natural duality, endodualisable algebra, endoprimal algebra.

The authors wish to express their gratitude to B.A. Davey and T. Katriňák for their helpful remarks and to J.G. Pitkethly for her assistance with the present pictures. A support by Slovak grants VEGA 1/4057/97, 1/3026/06 and APVV-51-009605 is acknowledged by the first author who also wishes to thank the Mathematical Institute of the University of Oxford and the School of Mathematical and Statistical Sciences of La Trobe University for their hospitality. 
on the quasivariety $\mathbb{I S P}(\mathbf{M})$, in the sense defined in [9]; it fails to be $k$-endoprimal if End $\mathbf{M}$ fails to yield a duality on the particular algebra $\mathbf{F}_{\mathbb{I S P}(\mathbf{M})}(k)$ (see [26], Proposition 2). The relationship between endodualisability and endoprimality and $k$-endoprimality has been explored, successively, in [15], [14], 17] and [26]. In the course of these investigations, examples drawn from varieties of algebras with lattice or semilattice reducts have provided valuable insights. Indeed, it was in the course of our investigations of endoprimal double Stone algebras that we found the results presented in the companion paper 26. There we proved the following general theorem.

Theorem 1.1. Let $\mathbf{D}$ be a finite algebra which is dualisable via the structure $\underset{\mathbf{D}}{\mathbf{D}}=(D ;$ End $\mathbf{D}, S, \tau)$ where $S$ is a finite set of finitary algebraic relations on $\widetilde{\mathbf{D}}$ and $\tau$ is the discrete topology. Assume there is a k-generated free algebra $\mathbf{F}_{\mathcal{D}}(k) \in \mathcal{D}=\mathbb{I S P}(\mathbf{D})$ which has each of the algebras $\mathbf{s} \in S$ as a retract. Let $\mathbf{M} \in \mathcal{D}$ be a finite algebra which has $\mathbf{D}$ as a retract. Then the following are equivalent:

(1) $\mathbf{M}$ is endoprimal;

(2) $\mathbf{M}$ is k-endoprimal;

(3) $\mathbf{M}$ is endodualisable.

This theorem was shown to apply, in particular, to any Stone algebra which is not Boolean, with $k=2$; further, the endoprimal algebras can be seen to be exactly those which have the four-element chain algebra as a retract. For double Stone algebras Theorem 1.1 does not generally apply. Nevertheless, we shall prove that the equivalence of (1)-(3), with either $k=3$ or $k=1$, does hold for each finite nonBoolean double Stone algebra $\mathbf{M}$, and the endoprimal algebras can be characterised in terms of retracts. A full statement of our results is given in Section 6 .

We recall that an algebra $\mathbf{L}=\left(L ; \vee, \wedge,{ }^{\star},{ }^{+}, 0,1\right)$ is called a double Stone algebra if $\mathbf{L}=\left(L ; \vee, \wedge,^{\star}, 0,1\right)$ and $\mathbf{L}=\left(L ; \wedge, \vee,{ }^{+}, 1,0\right)$ are Stone algebras, that is, if $\mathbf{L}$ is a bounded distributive lattice equipped with the pseudocomplementation operation * defined by

$$
x^{\star}=\max \{y \mid x \wedge y=0\}
$$

and with the dual pseudocomplementation operation ${ }^{+}$defined dually by

$$
x^{+}=\min \{y \mid x \vee y=1\},
$$

and if $\mathbf{L}$ satisfies the identities

$$
x^{\star} \vee x^{\star \star}=1 \text { and } x^{+} \wedge x^{++}=0 .
$$

Double Stone algebras form a variety $\mathcal{D S}=\mathbb{I S P}(4)$ generated by the four-element chain algebra

$$
\mathbf{4}=\left(\{0, a, b, 1\} ; \vee, \wedge,^{\star},{ }^{+}, 0,1\right)
$$

where $0<a<b<1$ and

$$
1^{\star}=b^{\star}=a^{\star}=0,0^{\star}=1,0^{+}=a^{+}=b^{+}=1,1^{+}=0 .
$$

The variety $\mathcal{D} \mathcal{S}$ of double Stone algebras is set in context as a variety of distributivelattice-ordered algebras in [10] and [34]. The non-trivial subdirectly irreducible 
double Stone algebras are the four-, three- and two-element chain algebras 4, $\mathbf{3}$ and $\mathbf{2}$, respectively. Consequently (see [28]) the variety $\mathcal{D S}$ has four subvarieties

$$
\mathfrak{T} \subset \mathcal{B} \subset \mathcal{R} \subset \mathcal{D S}
$$

where $\mathcal{T}$ is the subvariety of trivial algebras defined by $x=y, \mathcal{B}=\mathbb{I S P}(\mathbf{2})$ is the subvariety of Boolean algebras defined by $x^{\star}=y^{+}$and $\mathcal{R}=\mathbb{I S P}(\mathbf{3})$ is the subvariety of regular double Stone algebras defined by $x \vee x^{\star} \geqslant y \wedge y^{+}$. Algebras belonging to $\mathbb{I S P}(\mathbf{3})$ are also called three-valued Eukasiewicz algebras in the literature. Algebras belonging to $\mathbb{I S P}(\mathbf{4}) \backslash \mathbb{I S P}(3)$ will be called proper double Stone algebras in this paper.

The core of an algebra $\mathbf{L}$ in $\mathcal{D S}$ is defined to be

$$
K(\mathbf{L})=\left\{x \in L \mid x^{\star}=0, x^{+}=1\right\} ;
$$

this is non-empty if and only if $\mathbf{L}$ does not have $\mathbf{2}$ as a factor (see Section 2 ). When it is non-empty, the behaviour of the subset $K(\mathbf{L})$ in certain respects governs the behaviour of $\mathbf{L}$.

Repeatedly in the study of double Stone algebras it proves necessary to consider separately the cases when a given algebra is or is not regular and whether it has non-empty core or empty core. Below we relate these dichotomies to membership or non-membership of particular subquasivarieties of $\mathcal{D S}$. By setting up natural dualities for these subquasivarieties we are able to apply the techniques developed in [14] and 26].

\section{Double Stone algebras and their Priestley duality}

In this section we analyse finite double Stone algebras and record some elementary results on the structure of such algebras. A number of these are certainly well known, but for ease of reference we outline the proofs. The restricted Priestley duality theory which is available for finite double Stone algebras is particularly simple to work with, and we use it extensively. (We remark that this duality is, in fact, essentially the same as the natural duality for $\mathcal{D S}$ which appears in Section 4.)

We use boldface symbols to denote algebras, and the corresponding italic symbols to denote the underlying sets. Since it would be cumbersome to do otherwise we shall often abuse notation by using the same boldface symbol to denote both a double Stone algebra and its (bounded) distributive lattice reduct; which is meant will be clear from the context.

We shall assume familiarity with Priestley duality for bounded distributive lattices. A textbook summary can be found in [18; ; a presentation in the context of natural dualities appears in Chapter 1 of $[9]$. We denote by $\mathcal{D}$ the category of bounded distributive lattices $\mathbf{L}=(L ; \wedge, \vee, 0,1)$ and by $\mathcal{P}$ the dual category of compact totally order-disconnected spaces $(Y ; \leq, \mathcal{T})$ (Priestley spaces). We let $H: \mathcal{D} \rightarrow \mathcal{P}$ and $K: \mathcal{P} \rightarrow \mathcal{D}$ denote the adjoint functors setting up the dual equivalence between $\mathcal{D}$ and $\mathcal{P}$. The dual space $H(\mathbf{L})$ of $\mathbf{L} \in \mathcal{D}$ may be taken to be the prime filters of $\mathbf{L}$, ordered by inclusion and suitably topologised. The duality allows us to identify $\mathbf{L} \in \mathcal{D}$ with the lattice of clopen upsets (= order filters) of its 
dual space $H(\mathbf{L}) \in \mathcal{P}$. In case $L$ is finite the dual space of $\mathbf{L}$ is also finite, and is given by the join-irreducible elements of $\mathbf{L}$ with the reverse of the induced order. Its topology $\mathcal{T}$ is the discrete topology (which we always denote by $\tau$ ). Consequently in the finite case the topology plays no role and may be suppressed. For algebras which have a bounded distributive lattice reduct we may hope to set up a duality in which the additional operations are captured by additional structure on the dual spaces. One of the earliest such restricted Priestley dualities was that for Stone algebras, described in 32 . We now recall how this works for finite algebras.

Let $\mathbf{L}=(L ; \wedge, \vee, 0,1)$ be a finite member of $\mathcal{D}$. Identify $\mathbf{L}$ with the lattice of upsets of the poset $Y=H(\mathbf{L})$. For any finite poset $Y$, let $\operatorname{Max} Y$ and $\operatorname{Min} Y$ denote respectively the maximal and minimal points of $Y$; let $Y^{0}=Y \backslash(\operatorname{Max} Y \cup \operatorname{Min} Y)$. The operators $\downarrow$ and $\uparrow$ have their usual order-theoretic meanings (see [18], p. 14). The pseudocomplement of $x \in \mathbf{L}$ is given by the upset $Y \backslash \downarrow x$ (see, for example, [18, p. 205). The Stone algebra identity holds precisely when each point $p$ of $Y$ is majorised by a unique point, $g(p)$, of $\operatorname{Max} Y$ (continuity of the map $g$, which is needed in general, is automatic when $L$ is finite). We have here just the well-known fact that Stone algebras have the characteristic property that every prime filter is contained in a unique maximal prime filter. When this is the case we have for all upsets $x \in \mathbf{L}$

(i) $x^{\star}=Y \backslash \downarrow x=Y \backslash g^{-1}(x)$,

(ii) $x^{\star \star}=\downarrow x$, and this set is both an upset and a downset.

From this it follows immediately that the lattice filter

$$
D(\mathbf{L}):=\left\{x \in L \mid x^{\star}=0\right\}=\left\{x \vee x^{\star} \mid x \in L\right\}
$$

consists of those upsets which contain $\operatorname{Max} Y$.

Dual statements hold in respect of ${ }^{+}$. To see how, observe that the order-dual lattice $(L ; \wedge, \vee, 1,0)$ is identifiable with the downsets of $Y$, that is, the sets $Y \backslash x$, where $x$ is an upset. In particular, when $(L ; \wedge, \vee,+, 1,0)$ is a Stone algebra, each point $p$ of $Y$ majorises a unique minimal point $h(p)$ of $Y$ and

(i) $x^{+}=\uparrow(Y \backslash x)=Y \backslash h^{-1}(x)$,

(ii) $x^{++}=Y \backslash \uparrow(Y \backslash x)$.

Further, the ideal

$$
\bar{D}(\mathbf{L}):=\left\{x \in L \mid x^{+}=1\right\}=\left\{x \wedge x^{+} \mid x \in L\right\}
$$

consists of those upsets disjoint from Min $Y$. Observe that, for a finite double Stone algebra $\mathbf{L}$, the core $K(\mathbf{L})$ of $\mathbf{L}$ defined above is exactly $D(\mathbf{L}) \cap \bar{D}(\mathbf{L})$. We remark in passing that the variety $\mathcal{D} \mathcal{S}$ may be regarded as a subvariety of the variety of double $\mathcal{M} \mathcal{S}$-algebras. With this perspective the maps $g$ and $h$ are just the orderreversing maps on the dual spaces of such algebras which are customarily used to capture the negation operations ${ }^{\star}$ and ${ }^{+}$.

Given a finite poset $Y$, there is a finite double Stone algebra whose lattice reduct is isomorphic to the lattice of upsets of $Y$ if and only if each point $p$ lies below a unique maximal point $g(p)$ and above a unique minimal point $h(p)$. We shall call such posets $\mathcal{D} \mathcal{S}$-spaces (the word 'finite' is omitted here since we never study infinite Priestley dual spaces in this paper). Strictly, such spaces are structures of 
type $(Y ; \leq, g, h, \tau)$; since $g$ and $h$ are determined by the order we generally suppress them.

For each $n$, the $n$-element chain $\mathbf{n}$ is a $\mathcal{D S}$-space, dual to a double Stone algebra whose underlying lattice is a chain with $(n+1)$ elements. In general a finite poset $Y$ is a $\mathcal{D S}$-space if and only if it is the finite disjoint union of a (possibly empty) set of isolated points and a (possibly empty) family of posets of the form $\mathbf{1} \oplus Z \oplus \mathbf{1}$, where $Z$ is an arbitrary finite poset. In case $Z$ is an $n$-element antichain the associated algebra is $\mathbf{1} \oplus \mathbf{2}^{n} \oplus \mathbf{1}$, which we shall denote by $\mathbf{A}_{n}$. Under Priestley duality, products in the category of finite bounded distributive lattices correspond to disjoint unions in the category of finite posets. Because the maps $g$ and $h$ map each order component of the poset $Y$ into itself, products in $\mathcal{D S}$ of finite double Stone algebras are also given dually by disjoint unions.

Under Priestley duality, morphisms between finite bounded distributive lattices correspond, contravariantly, to order-preserving maps between the dual spaces. Explicitly, for a $\mathcal{D}$-homomorphism $f: \mathbf{L} \rightarrow \mathbf{M}$, the dual map $H(f): H(\mathbf{M}) \rightarrow H(\mathbf{L})$ is related to $f$ by $f=H(f)^{-1}$, where the lattices $\mathbf{L}$ and $\mathbf{M}$ are identified respectively with the upsets of the posets $H(\mathbf{L})$ and $H(\mathbf{M})$. Now let $\mathbf{L}$ and $\mathbf{M}$ be finite algebras in $\mathcal{D S}$. The map $f$ is ${ }^{\star}$-preserving if and only if its dual map $H(f)$ takes maximal points to maximal points. Likewise, $f$ preserves ${ }^{+}$if and only if $H(f)$ takes minimal points to minimal points.

The following easy lemma is central to our later investigations, as it enables us to describe the endomorphisms of any finite double Stone algebra and also the hom-sets which arise in connection with natural dualities. The conclusions of the lemma hold true when the variety $\mathcal{D S}$ is replaced by a variety $\mathcal{A}$ satisfying suitable general conditions, and with the algebras involved not restricted to be finite. In particular, it is sufficient to assume that $\mathcal{A}$ has factorisable congruences and that every subalgebra of an indecomposable algebra in $\mathcal{A}$ is also indecomposable. These conditions hold in particular when $\mathcal{A}=\mathfrak{D} \mathcal{S}$.

Lemma 2.1. Let $\mathbf{L}, \mathbf{M}$ be finite double Stone algebras and let $e \in \operatorname{hom}(\mathbf{L}, \mathbf{M})$. Assume that $\mathbf{L}=\mathbf{L}_{1} \times \cdots \times \mathbf{L}_{p}$, where $\mathbf{L}_{1}, \ldots, \mathbf{L}_{p}$ are indecomposable.

(i) Assume that $\mathbf{M}$ is indecomposable. Then there exist $j$ and a homomorphism $g: \mathbf{L}_{j} \rightarrow \mathbf{M}$ such that $e=g \circ \pi_{j}$, where $\pi_{j}: \mathbf{L} \rightarrow \mathbf{L}_{j}$ is the canonical projection.

(ii) Assume that $\mathbf{M}=\mathbf{M}_{1} \times \cdots \times \mathbf{M}_{q}$, where $\mathbf{M}_{1}, \ldots, \mathbf{M}_{q}$ are indecomposable. Then there exist a map $\epsilon:\{1, \ldots, q\} \rightarrow\{1, \ldots, p\}$ and maps $e_{i} \in \operatorname{hom}\left(\mathbf{L}_{\epsilon(i)}, \mathbf{M}_{i}\right)(i=1, \ldots, q)$ such that

$$
e\left(\left(a_{1}, \ldots, a_{p}\right)\right)=\left(e_{1}\left(a_{\epsilon(1)}\right), \ldots, e_{q}\left(a_{\epsilon(q)}\right)\right) \text { for all }\left(a_{1}, \ldots, a_{p}\right) \in \mathbf{L} \text {. }
$$

Proof. We prove (i). Part (ii) is then immediate from the definition of product.

The morphism $H(e)$ dual to $e$ is an order-preserving map $\theta$ from $H(\mathbf{M})$ to $H\left(\mathbf{L}_{1}\right) \cup \ldots \cup H\left(\mathbf{L}_{p}\right)$. The indecomposability assumptions ensure that the posets $H(\mathbf{M}), H\left(\mathbf{L}_{1}\right), \ldots, H\left(\mathbf{L}_{p}\right)$ are all connected. Any two points $y, z$ in $H(\mathbf{M})$ are joined by a fence, and the image of this fence under $\theta$ is a fence joining the image points $\theta(y), \theta(z)$. Consequently $\operatorname{im} \theta$ is connected and so lies in $H\left(\mathbf{L}_{j}\right)$ for some $j$. This gives (i). 
We next list properties of the core $K(\mathbf{L})$ of a finite double Stone algebra $\mathbf{L}$. We first remark that it is very easy to see algebraically that the core is either empty or is an interval $[a, b]$ in the lattice $\mathbf{L}$ (with $a$ the join of all atoms in $\mathbf{L}$ and $b$ the meet of all coatoms in $\mathbf{L}$ ). Alternatively, this fact can be derived from part (iv) of the lemma below. We note too that it is easy to prove algebraically or by duality that if $\mathbf{2}=\{0,1\}$ is a direct factor of $\mathbf{L}$ then $K(\mathbf{L})=\varnothing$ even when $\mathbf{L}$ is not finite. The converse is however not necessarily true. The characterisation of an algebra with a one-element core also does require finiteness.

Lemma 2.2. Let $\mathbf{L}$ be a finite double Stone algebra with dual space $Y$, and identify $L$ with the upsets of $Y$.

(i) $K(\mathbf{L})=\{x \in L \mid \operatorname{Max} Y \subseteq x \subseteq(Y \backslash \operatorname{Min} Y)\}$.

(ii) $|K(\mathbf{L})|=1$ if and only if $Y$ is the disjoint union of $\operatorname{Max} Y$ and $\operatorname{Min} Y$, the unique element of $K(\mathbf{L})$ then being the upset $\operatorname{Max} Y$.

(iii) The following are equivalent:

(a) $K(\mathbf{L})=\varnothing$

(b) $\operatorname{Max} Y \cap \operatorname{Min} Y \neq \varnothing$,

(c) $\mathbf{2}$ is a direct factor of $\mathbf{L}$.

(iv) If $K(\mathbf{L}) \neq \varnothing$ then the map $a \mapsto a \cap Y^{0}$ is a lattice isomorphism between $K(\mathbf{L})$ and the lattice of upsets of $Y^{0}$.

Proof. Assertion (i) is immediate from the dual descriptions of $D(\mathbf{L})$ and $\bar{D}(\mathbf{L})$, and (ii) and the equivalence of (a) and (b) in (iii) follow directly from this.

We now show that, in (iii), (b) is equivalent to (c). By the observation above concerning products, $\mathbf{2}$ is a direct factor of $\mathbf{L}$ if and only if $Y$ contains a point $p$ which is an isolated point with respect to the order. Such isolated points are exactly those in $\operatorname{Max} Y \cap \operatorname{Min} Y$. This completes the proof of (iii), and (iv) then follows from this and (i).

Let $\mathbf{L}$ be a finite double Stone algebra. It is obvious that $\mathbf{L}$ may be decomposed as $\mathbf{L}=\mathbf{J} \times \mathbf{2}^{\ell}$, where $\ell \geq 0$ and $\mathbf{J}$ does not have $\mathbf{2}$ as a direct factor. (We adopt the convention that $\mathbf{A}^{0}$ is the trivial algebra, for any algebra $\mathbf{A}$.) Thus according to part (iii) of the lemma above, $\mathbf{L}$ is the product of a Boolean algebra with an algebra which is either trivial or has non-empty core.

We shall need to recognize when particular double Stone algebra chains occur as retracts of a given algebra. Let $\mathbf{L}, \mathbf{M}$ be finite double Stone algebras with dual spaces $Y, Z$ respectively. Observe that $\mathbf{M}$ is a $\mathcal{D} \mathcal{S}$-retract of $\mathbf{L}$ if and only if $Z$ is a retract of $Y$ in the category of $\mathcal{D} \mathcal{S}$-spaces. More precisely, this happens if and only if there exist maps $\varphi: Y \rightarrow Z$ (retraction) and $\psi: Z \rightarrow Y$ (co-retraction), where $\varphi \circ \psi=\operatorname{id}_{Z}$ and $\varphi, \psi$ preserve the order and send maximal (minimal) points to maximal (minimal) points. We may assume without loss of generality that $Z$ is a subposet of $Y$.

Lemma 2.3. Let $\mathbf{L}$ be a finite non-trivial double Stone algebra with dual space $Y$.

(i) Assume that $K(\mathbf{L})=\varnothing$. Then $\mathbf{L}$ does not have $\mathbf{n}$ as a retract for any $n>2$.

(ii) Assume that $K(\mathbf{L}) \neq \varnothing$ (equivalently, $\operatorname{Max} Y \cap \operatorname{Min} Y=\varnothing)$.

(a) $\mathbf{3}$ is a retract of $\mathbf{L}$ always. 
(b) $\mathbf{4}$ is a retract of $\mathbf{L}$ if and only if $Y^{0} \neq \varnothing$.

(c) $\mathbf{5}$ is a retract of $\mathbf{L}$ if and only if $Y^{0}$ is not an antichain.

Proof. Note that $Y \neq \varnothing$ since $\mathbf{L}$ is assumed to be non-trivial. Observe also that any morphism between $\mathcal{D} \mathcal{S}$-spaces maps any point which is simultaneously maximal and minimal to a point with the same property. Therefore, if $\operatorname{Max} Y \cap \operatorname{Min} Y \neq \varnothing$ there is no retraction of $Y$ onto a chain with at least two points. This proves (i).

Now assume $\operatorname{Max} Y \cap \operatorname{Min} Y=\varnothing$. We need conditions under which $Y$ retracts onto $\mathbf{n}(n=2,3,4)$. First consider $n=2$. Take a two-element chain $\{p, q\}$ in $Y$ with $p \in \operatorname{Min} Y$ and $q \in \operatorname{Max} Y$. We can retract $Y$ onto this chain: for the retraction we send $\operatorname{Min} Y$ to $\{p\}$ and $Y \backslash \operatorname{Min} Y$ to $\{q\}$ and for the co-retraction we take the map fixing $p$ and $q$. The cases $n=3$ and $n=4$ are similar. We can retract $Y$ onto a three-element subchain of $Y$ exactly when $Y$ contains a three-element chain. When this occurs there is a chain $p<w<q$ in $Y$ with $p \in \operatorname{Min} Y, q \in \operatorname{Max} Y$ and $w \in Y^{0}$, and we can map $\operatorname{Min} Y$ to $\{p\}, Y^{0}$ to $\{w\}$, and $\operatorname{Max} Y$ to $\{q\}$. Likewise, we can retract $Y$ onto a four-element subchain provided there is a four-element chain in $Y$. This condition holds if and only if $Y^{0}$ is not an antichain, and in this case there is a retraction, in the category of finite posets, of $Y^{0}$ onto a two-element subchain (see [15]). The required retraction is then easily constructed.

The following lemma will allow us to describe in terms of Priestley dual spaces the finite members of certain subquasivarieties of $\mathcal{D} \mathcal{S}$. An analogous result can be obtained without the finiteness restriction; see 9], 1.3.1 and 7.4.1(vi).

Lemma 2.4. Let $\mathfrak{C}$ be the class of finite algebras in a variety $\mathcal{D}$ of algebras of type $\mathbf{D}=(D ; \vee, \wedge, 0,1, F)$, where the reduct $(D ; \vee, \wedge, 0,1)$ is a finite bounded distributive lattice. Assume that $\mathbf{C}$ is dually equivalent under a restricted Priestley duality to a class $\boldsymbol{y}$ of finite posets with additional structure which is set up by dually adjoint functors $H: \mathcal{C} \rightarrow \boldsymbol{y}$ and $K: \boldsymbol{y} \rightarrow \mathcal{C}$. Let $\mathbf{M}$ be a finite algebra in $\mathcal{D}$ and let $\mathcal{A}=\mathbb{I S P}(\mathbf{M})$ be the quasivariety it generates. Then the following are equivalent for a finite algebra $\mathbf{A} \in \mathcal{A}$ with dual $H(\mathbf{A})$ :

(1) $\mathbf{A} \in \mathbb{I} \mathbb{S P}(\mathbf{M})$;

(2) for each $z \in H(\mathbf{A})$ there exists a $\mathbf{y}$-morphism $\theta: H(\mathbf{M}) \rightarrow H(\mathbf{A})$ such that $z \in \operatorname{im} \theta$.

Proof. It is clear that an algebra $\mathbf{A}$ in a variety $\mathcal{V}$ lies in the quasivariety $\operatorname{ISP}(\mathbf{M})$ generated by an algebra $\mathbf{M} \in \mathcal{V}$ if and only if, for any pair of distinct elements $c, d \in A$, there exists a homomorphism $u: \mathbf{A} \rightarrow \mathbf{M}$ such that $u(c) \neq u(d)$ (see for example [9], p. 16). Identify $\mathbf{A}$ with the lattice of upsets of $H(\mathbf{A})$. Assume (1) holds. Let $z \in H(\mathbf{A})$ and take the distinct upsets $c=\uparrow z$ and $d=\uparrow z \backslash\{z\}$. Take a homomorphism $u: \mathbf{A} \rightarrow \mathbf{M}$ with $u(c) \neq u(d)$. Obviously, the $\mathbf{y}$-morphism $\theta=H(u): H(\mathbf{M}) \rightarrow H(\mathbf{A})$ satisfies $u(x)=\theta^{-1}(x)$ for all $x \in \mathbf{A}$. If $z \notin \operatorname{im} \theta$ we would have $u(c)=u(d)$, contrary to hypothesis.

Conversely, assume that (2) holds, and let $c \neq d$ in A. Assume without loss of generality that $c \nsubseteq d$ and let $z \in c \backslash d$. Let $\theta: H(\mathbf{M}) \rightarrow H(\mathbf{A})$ be as in (2), and choose $p \in H(\mathbf{M})$ with $z=\theta(p)$. Let $u \in \operatorname{Hom}(\mathbf{A}, \mathbf{M})$ be given by $u:=\theta^{-1}$, so that $\theta=H(u)$. Now we have $p \in u(c) \backslash u(d)$, whence $u(c) \neq u(d)$. 


\begin{tabular}{|c|c|}
\hline $\mathbf{L}$ with Boolean core & $\begin{array}{l}Y \backslash(\operatorname{Max} Y \cup \operatorname{Min} Y) \text { an antichain } \\
K(\mathbf{L}) \neq \varnothing \text { and } \mathbf{5} \text { is not a retract of } \mathbf{L} \\
\mathbf{L} \text { is a product of algebras of the form } \mathbf{A}_{n} \\
\quad(n \geq 1)\end{array}$ \\
\hline $\mathbf{L} \in \mathbb{I S P P}(\mathbf{4} \times \mathbf{2})$ & $\begin{array}{l}\operatorname{Max} Y \cap \operatorname{Min} Y \neq \varnothing \\
K(\mathbf{L})=\varnothing \\
\mathbf{2} \text { is a factor of } \mathbf{L}\end{array}$ \\
\hline $\mathbf{L} \in \mathbb{I} \mathbb{S P}(\mathbf{3})$ & $\begin{array}{l}Y=\operatorname{Max} Y \cup \operatorname{Min} Y \\
|K(\mathbf{L})| \leq 1 \\
\mathbf{4} \text { is not a retract of } \mathbf{J} \\
\mathbf{L} \text { is of the form } \mathbf{3}^{m} \times \mathbf{2}^{\ell}(m, \ell \geq 0)\end{array}$ \\
\hline $\begin{array}{l}\mathbf{L} \in \mathbb{I S P P}(\mathbf{3} \times \mathbf{2})= \\
\quad \mathbb{I S P}(\mathbf{4} \times \mathbf{2}) \cap \mathbb{I S P}(\mathbf{3})\end{array}$ & $\begin{array}{l}Y=\operatorname{Max} Y \cup \operatorname{Min} Y \text { and } \operatorname{Max} Y \cap \operatorname{Min} Y \neq \varnothing \\
\mathbf{2} \text { is a factor of } \mathbf{L} \text { and } \mathbf{4} \text { is not a retract of } \mathbf{J} \\
\mathbf{L} \text { is of the form } \mathbf{3}^{m} \times \mathbf{2}^{\ell}(m \geq 0, \ell \geq 1)\end{array}$ \\
\hline $\begin{array}{l}\mathbf{L} \text { a Post algebra } \\
\text { of order } 3\end{array}$ & $\begin{array}{l}Y=\operatorname{Max} Y \cup \operatorname{Min} Y \\
|K(\mathbf{L})|=1 \\
\mathbf{L} \text { is of the form } \mathbf{3}^{m}(m \geq 1)\end{array}$ \\
\hline $\mathbf{L} \in \mathbb{I S P}(\mathbf{2})$ & $\begin{array}{l}Y=\operatorname{Max} Y=\operatorname{Min} Y \\
\mathbf{L} \text { is Boolean } \\
\mathbf{3} \text { is not a retract of } \mathbf{L} \\
\mathbf{L} \text { is of the form } \mathbf{2}^{\ell}(\ell \geq 0)\end{array}$ \\
\hline
\end{tabular}

Table 1

Applying this result to the variety $\mathcal{D} \mathcal{S}$, and, putting together the information contained in the preceding lemmas and discussion, we derive the characterisations contained in Table 1. In this table, $\mathbf{L}$ is to be taken to be a finite double Stone algebra, expressed as $\mathbf{L}=\mathbf{J} \times \mathbf{2}^{\ell}$, where $\ell \geq 0$ and $\mathbf{J}$ does not have $\mathbf{2}$ as a direct factor.

\section{Natural dualities: preliminaries}

The theory of natural dualities concerns the topological representation of algebras. The main idea is that, given a quasivariety $\mathcal{D}=\mathbb{I S P}(\mathbf{D})$ of algebras generated by an algebra $\mathbf{D}$, one can seek a topological relational structure $\mathbf{\sim}$ on the underlying set $D$ of $\mathbf{D}$ such that a dual equivalence exists between $\mathcal{D}$ and a suitable category $\boldsymbol{X}$ of topological relational structures of the same type as $\underset{\sim}{\mathbf{D}}$. As a result one can obtain a uniform way of representing each algebra $\mathbf{M}$ in the quasivariety $\mathcal{D}$ as an algebra of continuous structure-preserving maps from a dual $\mathbf{X} \in \mathcal{X}$ of the algebra $\mathbf{M}$ into $\underset{\sim}{\mathbf{D}}$.

More precisely, let $\mathbf{D}=(D ; F)$ be a finite algebra. Let $\underset{\sim}{\mathbf{D}}=(D ; R, \tau)$ be the set $D$ endowed with a (finite) set $R$ of relations and with the discrete topology $\tau$. The structure $\underset{\sim}{\mathbf{D}}$ is algebraic over $\mathbf{D}$ if each relation $r$ in $R$ is a subalgebra of 
an appropriate power of $\mathbf{D}$; in such a situation we write $\mathbf{r}$ to denote $r$, qua algebra. The graph of an $n$-ary operation $g: D^{n} \rightarrow D$ is the $(n+1)$-ary algebraic relation

$$
g^{\square}=\left\{\left(x_{1}, \ldots, x_{n}, g(\mathbf{x})\right) \mid\left(x_{1}, \ldots, x_{n}\right) \in D^{n}\right\} \subseteq D^{n+1} .
$$

Note, in particular, that the graph of any endomorphism $g \in$ End $\mathbf{D}$ is algebraic over D.

Let $\underset{\sim}{\mathbf{D}}=(D ; R, \tau)$ be algebraic over $\mathbf{D}$. Let $\boldsymbol{X}=\mathbb{I} \mathbb{S}_{c} \mathbb{P}(\underset{\sim}{\mathbf{D}})$ denote the class of all structures which are embeddable as closed substructures into powers of $\underset{\sim}{\mathbf{D}}$. Let $\mathbf{M} \in \mathcal{D}$. Let $D(\mathbf{M}):=\operatorname{hom}(\mathbf{M}, \mathbf{D})$ be the set of all $\mathcal{D}$-homomorphisms $\mathbf{M} \rightarrow \mathbf{D}$. With the relations of $R$ acting pointwise on $D(\mathbf{M})$ and the product topology of $\mathbf{D}^{M}$ restricted to $D(\mathbf{M})$, we can naturally interpret $D(\mathbf{M})$ as a substructure of $\mathbf{D}^{M}$, hence as a member of $\boldsymbol{X}$.

Let $r \subseteq D^{n}$. We let the relation $r$ act on the dual $D(\mathbf{M})$ as follows: if $h_{1}, \ldots, h_{n} \in D(\mathbf{M})$ are such that $\left(h_{1}(a), \ldots, h_{n}(a)\right) \in r$ for every $a \in M$ we write $\left(h_{1}, \ldots, h_{n}\right) \in r_{D(\mathbf{M})}$. We say that a map $u: D(\mathbf{M}) \rightarrow \mathbf{D}$ preserves the relation $r$ on $D(\mathbf{M})$ if $\left(u\left(h_{1}\right), \ldots, u\left(h_{n}\right)\right) \in r$ for every action $\left(h_{1}, \ldots, h_{n}\right) \in r_{D(\mathbf{M})}$ of $r$ on $D(\mathbf{M})$. Observe that, for any $e \in \operatorname{End} \mathbf{D}$, the map $u$ preserves the relation $e^{\square}$ on $D(\mathbf{M})$ if and only if $u(e \circ h)=e(u(h))$ for every $h \in D(\mathbf{M})$. This allows us, with a slight abuse of notation, to regard End $\mathbf{M}$ as a set of binary relations.

Consider again the dual $D(\mathbf{M})$ as a member of $\boldsymbol{X}$. A map $u: D(\mathbf{M}) \rightarrow \underset{\sim}{\mathbf{D}}$ is called a morphism if it is continuous and preserves all relations in $R$. Denote by $E(\mathbf{X}):=\operatorname{hom}(\mathbf{X}, \underset{\sim}{\mathbf{D}})$ the set of all morphisms from $D(\mathbf{M})$ into $\underset{\sim}{\mathbf{D}}$. Then $E(D(\mathbf{M}))$ can be naturally understood as a subalgebra of $\mathbf{D}^{D(\mathbf{M})}$, that is, a member of $\mathcal{D}$. The (hom-)functors $D: \mathcal{D} \rightarrow \mathcal{X}$ and $E: \mathcal{X} \rightarrow \mathcal{D}$ are contravariant and dually adjoint. Moreover, for any $\mathbf{M} \in \mathcal{D}$ we have a natural morphism $e_{M}: \mathbf{M} \rightarrow E D(\mathbf{M})$ given by evaluation, viz.

$$
e_{M}(a)(h)=h(a) \text { for every } a \in M \text { and } h \in D(\mathbf{M}),
$$

which is an embedding. Let $\underset{\sim}{\mathbf{D}}=(D ; R, \tau)$ be algebraic over $\mathbf{D}$. We say that $\underset{\sim}{\mathbf{D}}$ (or just $R$ ) yields a (natural) duality on an algebra $\mathbf{M} \in \mathcal{D}$ if the embedding $e_{M}$ is an isomorphism, that is, the evaluation maps $e_{M}(a)(a \in M)$ are the only morphisms from $D(\mathbf{M})$ to $\mathbf{D}$. We say that $\underset{\sim}{\mathbf{D}}$ (or $R$ ) yields a (natural) duality on the quasivariety $\mathcal{D}$ if it yields a duality on every $\mathbf{M} \in \mathcal{D}$. Assume this is so. Then if $R=$ End $\mathbf{D}$ we say that $\mathbf{D}$ is endodualisable and if $R=$ End $D \cup\{s\}$ we say that $\mathbf{D}$ is almost endodualisable (with extra relation $s$ ).

Endoprimality is linked to duality theory by a result based on folklore and recorded explicitly in 26 .

Proposition 3.1. Let $\mathbf{M}$ be a finite algebra and let $\mathcal{A}=\mathbb{I S P}(\mathbf{M})$. Then $\mathbf{M}$ is $k$ endoprimal if and only if $\operatorname{End}(\mathbf{M})$ yields a duality on the free algebra $\mathbf{F}_{\mathcal{A}}(k)$ and is therefore endoprimal if and only if $\operatorname{End}(\mathbf{M})$ yields a duality on all the free algebras $\mathbf{F}_{\mathcal{A}}(k)(k \geq 1)$. Further, $\mathbf{M}$ is endodualisable implies that $\mathbf{M}$ is endoprimal.

Suppose we wish to decide whether a given finite algebra $\mathbf{M}$ is endodualisable. Our aim will be to find a dualising structure $\mathbf{M}$ for $\mathbf{M}$ which contains End $\mathbf{M}$ together with an additional set $S$ of algebraic relations. We shall then seek to 
show that the elements of $S$ can be discarded without destroying the duality; in that case $\mathbf{M}$ is endodualisable. Alternatively, we may seek to show that $\mathbf{M}$ is not endodualisable, by exhibiting an algebra $\mathbf{A}$ in $\mathbb{I S P}(\mathbf{M})$ on which End $\mathbf{M}$ does not yield a duality. More precisely, if there are $\mathbf{A} \in \mathbb{I S P}(\mathbf{M})$, a map $u: D(\mathbf{A}) \rightarrow M$ preserving End $\mathbf{M}$, an algebraic relation $r$ on $M$ and $z_{1}, \ldots, z_{n} \in D(\mathbf{A})$ such that $\left(z_{1}, \ldots, z_{n}\right) \in r_{D(\mathbf{A})}$, but $\left(u\left(z_{1}\right), \ldots, u\left(z_{n}\right)\right) \notin r$, we shall say that $(\mathbf{A}, r)$ is a test pair, with a test algebra $\mathbf{A}$ and a test relation $r$, witnessed by $u$, showing that End $\mathbf{M}$ does not yield a duality on $\mathcal{A}$. An obvious candidate for such a test algebra $\mathbf{A}$ is $\mathbf{s}$, for some $s \in S$. This key observation, originating in 20, has a significant converse, the Test Algebra Lemma ([20, Proposition 2.3). This can be used to identify relations in a dualising structure which are superfluous. Combined with [22], Lemmas 4.1 and 4.2 it gives the following lemma.

Lemma 3.2. Let $\mathbf{M}$ be a finite algebra and assume that $\mathbf{M}=(M$, End $M, S, \tau)$ yields a duality on $\mathbb{I S P}(\mathbf{M})$. Then any relation $s \in S$ which is such that $\mathbf{s}$ is isomorphic to a retract of $\mathbf{M}$ can be deleted from $S$ without destroying the duality.

These ideas are pursued further in [26] to give the Retraction Test Algebra Lemma, which underlies Theorem 1.1. Informally stated, the lemma says that any algebra which retracts onto a known test algebra also serves as a test algebra. In conjunction with Proposition 3.1 it has the following consequence.

Proposition 3.3. ([26], Corollary 7) Let $\mathbf{M}$ be a finite algebra. Assume that $\mathbf{M}$ is not endodualisable and that this is shown by a test algebra $\mathbf{s}$ in $\mathcal{A}=\mathbb{I S P}(\mathbf{M})$. Assume further that $\mathbf{s}$ is a retract of the $k$-generated free algebra $\mathbf{F}_{\mathcal{A}}(k)$. Then $\mathbf{M}$ is not k-endoprimal.

This proposition is, of course, of little value if we do not know the free algebras in $\operatorname{ISP}(\mathbf{M})$. We recall the following remarks from $[26$. If $\mathbf{M}$ above is a finite algebra in $\mathcal{D}=\mathbb{I S P}(\mathbf{D})$ such that $\mathbf{M}$ has $\mathbf{D}$ as a retract, then $\mathcal{A}=\mathcal{D}$ and the $k$-generated free algebras $\mathbf{F}_{\mathcal{A}}(k)$ and $\mathbf{F}_{\mathcal{D}}(k)$ are (up to isomorphism) the same. To apply Proposition 3.3 for such a non-endodualisable algebra $\mathbf{M}$ with a test algebra $\mathbf{s}$, it is therefore sufficient to decide whether $\mathbf{s}$ is a retract of one of the free algebras $\mathbf{F}_{\mathcal{D}}(k)$ in $\mathcal{D}$.

In order to identify that a finite algebra $\mathbf{M}$ is endodualisable we first need to find a duality for $\mathbb{I S P}(\mathbf{M})$. Frequently $\mathbf{M}$ will be a member of some given quasivariety $\operatorname{ISP}(\mathbf{D})$, for which a dualising structure $\mathbf{D}$ is already known. Provided $\mathbf{M}$ has $\mathbf{D}$ as a retract we can 'lift up' the structure $\underset{\sim}{\mathbf{D}}$ to find a dualising structure $\mathbf{M}$. This method, first employed by B.A. Davey in [14, is made precise in the following theorem; compare with Proposition 3.5(i) below and see [17, Theorems 1.5 and 1.6 and [26], Theorem 2.

Theorem 3.4. Let $\mathbf{D}$ be a finite algebra. Let $\mathbf{M}$ be a finite algebra in $\operatorname{ISP}(\mathbf{D})$ and assume that $\mathbf{D}$ is a retract of $\mathbf{M}$. If $\mathbf{D}$ is dualised via $\underset{\sim}{\mathbf{D}}=(D ;$ End $\mathbf{D}, s, \tau)$, with $s$ a finitary algebraic relation on $\mathbf{D}$, then $\mathbf{M}$ is dualised via $\underset{\sim}{\mathbf{M}}=\left(M ;\right.$ End $\left.\mathbf{M}, s^{\prime}, \tau\right)$ where $s^{\prime}$ is an algebraic relation on $M$ such that $s^{\prime}$ is of the same arity as $s$ and $\mathbf{s}^{\prime}$ is isomorphic to $\mathbf{s}$. If, further, $\mathbf{s}$ is (isomorphic to) a retract of $\mathbf{M}$ then $\mathbf{M}$ is endodualisable. 
In the same spirit as Theorem 3.4 is the result which follows. Parts (i) and (iii) are from [17. In the proof of part (i) (see also 35]) it is shown that End $\mathbf{M}$ yields a duality on all algebras $\mathbf{A} \in \mathcal{D}$ provided End $\mathbf{D}$ does. In exactly the same way the statement (ii) below can be proved for an (arbitrary) single algebra $\mathbf{A} \in \mathcal{D}$. Then (iii) is just a reformulation of (ii) for $\mathbf{A}=\mathbf{F}_{\mathcal{D}}(k)$ (see Proposition 3.1). In [17, (iii) has a purely algebraic proof, which does not require $\mathbf{M}$ to be finite.

Theorem 3.5. (cf. [17, Theorems 1.5 and 1.2) Let $\mathbf{D}$ be a finite algebra. Let $\mathbf{M}$ be an algebra in $\mathcal{D}=\mathbb{I S P}(\mathbf{D})$ and assume that $\mathbf{D}$ is a retract of $\mathbf{M}$.

(i) Assume $\mathbf{M}$ is finite. If $\mathbf{D}$ is endodualisable then so is $\mathbf{M}$.

(ii) Assume $\mathbf{M}$ is finite and $\mathbf{A} \in \mathbb{I S P}(\mathbf{M})$. If End $\mathbf{D}$ yields a duality on the single algebra $\mathbf{A}$ then End $\mathbf{M}$ does so.

(iii) If $\mathbf{D}$ is $k$-endoprimal then so is $\mathbf{M}$.

We deduce that a finite power of a finite endodualisable ( $k$-endoprimal) algebra $\mathbf{D}$ is endodualisable ( $k$-endoprimal). We now prove a partial converse, by making an assumption on the form taken by homomorphisms from $\mathbf{D}^{n}$ to $\mathbf{D}$; by Lemma 2.1, this assumption is satisfied when $\mathbf{D}$ is a finite indecomposable double Stone algebra. We note that essentially the same result as in (i) was obtained by M. Saramago 35] using a different proof.

Theorem 3.6. Assume that $\mathbf{D}$ is a finite algebra such that every subalgebra of $\mathbf{D}$ is indecomposable and that every homomorphism from $\mathbf{D}^{n}$ to $\mathbf{D}$ is expressible in the form $e \circ \rho_{i}$, for some projection map $\rho_{i}: \mathbf{D}^{n} \rightarrow \mathbf{D}$ and some $e \in$ End $\mathbf{D}$. Let $n>1$.

(i) If $\mathbf{D}$ is not endodualisable then neither is $\mathbf{D}^{n}$.

(ii) If $\mathbf{D}$ is not $k$-endoprimal then neither is $\mathbf{D}^{n}$. Specifically, if $\mathbf{D}$ is shown to fail to be $k$-endoprimal by a function $f: D^{k} \rightarrow D$, then $\mathbf{D}^{n}$ is shown to fail to be $k$-endoprimal by the co-ordinatewise lifting of $f$ to a function from $\left(D^{n}\right)^{k}$ to $D^{n}$.

Proof. Consider (i). We assume that there exists a test pair $(\mathbf{A}, r)$, witnessed by a map $\varphi$, showing that $\mathbf{D}$ is not dualised by End $\mathbf{D}$. Specifically, $\mathbf{A}$ is some algebra in $\mathbb{I S P}(\mathbf{D}), r$ is an algebraic relation on $\mathbf{D}$ and $\varphi: \operatorname{hom}(\mathbf{A}, \mathbf{D}) \rightarrow \mathbf{D}$ preserves End $\mathbf{D}$ but fails to preserve $r$.

Observe that $\mathbb{I S P}(\mathbf{D})=\mathbb{I} \mathbb{S P}\left(\mathbf{D}^{n}\right)$. Therefore $\mathbf{A}$ lies in the quasivariety $\mathcal{D}$ generated by $\mathbf{D}^{n}$. Also, given the $m$-ary algebraic relation $r$ over $\mathbf{D}$ there is an associated $m$-ary relation $r^{\Delta}$ which is algebraic over $\mathbf{D}^{n}$. This is defined by

$$
\left(a_{1}, \ldots, a_{m}\right) \in r^{\Delta} \Longleftrightarrow\left(\rho_{i}\left(a_{1}\right), \ldots, \rho_{i}\left(a_{m}\right)\right) \in r \text { for } i=1, \ldots, n .
$$

We claim there is a test pair $\left(\mathbf{A}, r^{\Delta}\right)$ witnessed by a map $\psi: \operatorname{hom}\left(\mathbf{A}, \mathbf{D}^{n}\right) \rightarrow \mathbf{D}^{n}$ showing that End $\mathbf{D}^{n}$ does not dualise $\mathbf{D}^{n}$. We define the map $\psi$ by

$$
\psi(x)=\left(\varphi\left(\rho_{1} \circ x\right), \ldots, \varphi\left(\rho_{n} \circ x\right)\right),
$$

where $x \in \operatorname{hom}\left(\mathbf{A}, \mathbf{D}^{n}\right)$ and $\rho_{1}, \ldots, \rho_{n}$ denote the projection maps of $\mathbf{D}^{n}$ onto $\mathbf{D}$. We will first show that $\psi$ preserves End $\mathbf{D}^{n}$, that is, that $\psi(e \circ x)=e(\psi(x))$ for 
each $x \in \operatorname{hom}\left(\mathbf{A}, \mathbf{D}^{n}\right)$ and $e \in$ End $\mathbf{D}^{n}$. By the assumption in the statement of the proposition we may write

$$
e\left(\left(a_{1}, \ldots, a_{n}\right)\right)=\left(e_{1}\left(a_{\epsilon(1)}\right), \ldots, e_{n}\left(a_{\epsilon(n)}\right)\right),
$$

where $\epsilon:\{1, \ldots, n\} \rightarrow\{1, \ldots, n\}$ and $e_{1}, \ldots, e_{n} \in$ End $\mathbf{D}$. We have, for any $b \in \mathbf{A}$,

$$
\begin{aligned}
(e \circ x)(b) & =e\left(\left(\rho_{1} \circ x\right)(b), \ldots,\left(\rho_{n} \circ x\right)(b)\right) \\
& =\left(\left(e_{1} \circ \rho_{\epsilon(1)} \circ x\right)(b), \ldots,\left(e_{n} \circ \rho_{\epsilon(n)} \circ x\right)(b)\right) .
\end{aligned}
$$

Therefore

$$
\begin{aligned}
\psi(e \circ x) & =\left(\varphi\left(e_{1} \circ \rho_{\epsilon(1)} \circ x\right), \ldots, \varphi\left(e_{n} \circ \rho_{\epsilon(n)} \circ x\right)\right) \\
& =\left(e_{1}\left(\varphi\left(\rho_{\epsilon(1)} \circ x\right)\right), \ldots, e_{n}\left(\varphi\left(\rho_{\epsilon(n)} \circ x\right)\right)\right) \\
& =e\left(\left(\varphi\left(\rho_{1} \circ x\right), \ldots, \varphi\left(\rho_{n} \circ x\right)\right)\right) \\
& =e(\psi(x))
\end{aligned}
$$

as required.

Given any $y \in \operatorname{hom}(\mathbf{A}, \mathbf{D})$ there exists $x \in \operatorname{hom}\left(\mathbf{A}, \mathbf{D}^{n}\right)$ such that $\rho_{i} \circ x=y$ for all $i$, where $x=y \sqcap \cdots \sqcap y$ (that is, $x(b):=(y(b), \ldots, y(b))$ for all $b \in \mathbf{A})$. Write $x$ as $y^{\Delta}$. Assume that the test relation $r$ is $m$-ary and that $\left(y_{1}, \ldots, y_{m}\right) \in r_{\text {hom }(\mathbf{A}, \mathbf{D})}$ but $\left(\varphi\left(y_{1}\right), \ldots, \varphi\left(y_{n}\right)\right) \notin r$. The definitions imply that $\left(y_{1}{ }^{\Delta}, \ldots, y_{m}{ }^{\Delta}\right) \in\left(r^{\Delta}\right)_{\operatorname{hom}\left(\mathbf{A}, \mathbf{D}^{n}\right)}$ but $\left(\psi\left(y_{1}{ }^{\Delta}\right), \ldots, \psi\left(y_{m}{ }^{\Delta}\right)\right) \notin r^{\Delta}$.

For (ii) we use the same proof, taking the test algebra $\mathbf{A}$ to be the $k$-generated free algebra in $\mathbb{I S P}(\mathbf{D})$ (which equals $\mathbb{I S P}\left(\mathbf{D}^{n}\right)$ ). Now consider the final assertion. We use, in the manner described in [26], the natural bijection between $D^{k}$ and the dual $D\left(F_{\mathbb{I S P}(\mathbf{D})}(k)\right)$ in $\operatorname{ISP}(\mathbf{D})$ of the algebra $\mathbf{A}$, and similarly between $\left(D^{n}\right)^{k}$ and the dual $D\left(F_{\mathbb{I S P}\left(\mathbf{D}^{n}\right)}(k)\right)$ in $\mathbb{I S P}\left(\mathbf{D}^{n}\right)$ of the algebra $\mathbf{A}$. Under the identifications these bijections allow, we may regard $\varphi$ as a function from $D^{k}$ to $D$ which preserves End $\mathbf{D}$ but is not a term function, and its co-ordinatewise lifting $\psi$ as a function from $\left(D^{n}\right)^{k}$ to $D^{n}$ which preserves End $\mathbf{D}^{n}$ but is not a term function.

\section{Dualities for subquasivarieties of double Stone algebras and endodu- alisability}

We now want to set up natural dualities for the variety $\mathcal{D} \mathcal{S}$ and the subquasivarieties of it arising in Table 1.

It is well known that, given any finite lattice-ordered algebra $\mathbf{D}$, we may always construct a duality for $\mathbb{I S P}(\mathbf{D})$ by taking $R=\mathbb{S}\left(\mathbf{D}^{2}\right)$, by the NU Duality Theorem (see, for example, [9], Chapter 4). We could use this 'brute force' method on our selected quasivarieties. This involves describing the subalgebras of $\mathbf{D}^{2}$ and then working out which of these are entailed by End $\mathbf{D}$, in the sense defined in 16] (or see 9, Chapter 8). This is indeed feasible, but the calculations are quite complicated. Instead of taking this route we opt for finding a more economical duality at the 
outset. To do this, we employ the piggyback technique, as described in [19] and 9], Chapter 7.

Proposition 4.1. (Piggyback Duality Theorem, for distributive-lattices-ordered algebras) Suppose that $\mathcal{D}=\mathbb{I S P}(\mathbf{D})$, where $\mathbf{D}$ is a finite algebra having a bounded distributive lattice reduct with Priestley dual $Z$. Let $\Omega$ be a fixed subset of $Z$.

Let $\mathbf{D}=(D ; R, \tau)$ be the topological relational structure in which

(i) $\tau$ is the discrete topology,

(ii) $R=$ End $\mathbf{D} \cup S$, where $S$ is the collection of maximal $\mathcal{D}$-subalgebras of sublattices of the form

$$
(\alpha, \beta)^{-1}(\leq):=\left\{(a, b) \in D^{2} \mid \alpha(a) \leq \beta(b)\right\},
$$

where $\alpha, \beta$ range over $\Omega$.

Let $\boldsymbol{X}:=\mathbb{I} \mathbb{S}_{c} \mathbb{P}(\underset{\sim}{\mathbf{D}})$. Assume that the following separation condition $(\mathrm{S})$ is satisfied:

(S) given $a, b \in D$ with $a \neq b$, there exist $u \in$ End $\mathbf{D}$ and $\alpha \in \Omega$ such that $\alpha(u(a)) \neq \alpha(u(b))$.

Then $\underset{\sim}{\mathbf{D}}$ yields a natural duality on $\mathcal{D}$.

The condition (S) can always be satisfied by taking $\Omega=Z$, but in practice we want to choose $\Omega$ to be as small as possible, to minimise the size of $S$ and hence the complexity of the resulting duality. Theorem 4.1 is applicable in particular to algebras in which the operations $F$ in $\mathbf{D}=(D ; \vee, \wedge, 0,1, F)$ are endomorphisms or dual endomorphisms of $\mathbf{D}$. Algebras of this type are called Cornish algebras in 25] in recognition of the contribution made by W.H. Cornish in providing a unified framework for their theory (see [10]). Natural duality theory for quasivarieties generated by Cornish algebras in general is worked out in [34].

In order to apply Theorem 4.1 to a given quasivariety $\mathbb{I S P}(\mathbf{D})$ we have to do two things: we first want to choose $\Omega$ in as economical a way as possible consistent with satisfying (S) and we must then identify the subalgebras associated with pairs $\alpha, \beta \in \Omega$. For the first of these tasks we employ the following lemma. This is proved using similar ideas to those needed in the proof of Lemma 2.4.

Lemma 4.2. Let $\mathbf{D}, R, Z, \Omega$ and condition (S) be as prescribed in Theorem 4.1. Assume also $\boldsymbol{y}$ and $H: \mathcal{D} \rightarrow \boldsymbol{y}$ are as in Lemma 2.4. Then (S) is satisfied so long as

$$
Z=\{(H(u))(\alpha) \mid u \in \text { End } \mathbf{D}, \alpha \in \Omega\} .
$$

We now apply this to double Stone algebras.

Lemma 4.3. Assume that $Z$ is a finite $\mathcal{D} \mathcal{S}$-space dual to a non-trivial and nonBoolean algebra $\mathbf{D} \in \mathcal{D S}$.

(i) Assume that $Z^{0} \neq \varnothing$. Then (S) is satisfied by taking $\Omega=\{\alpha\}$, where $\alpha \in Z^{0}$.

(ii) Assume that $Z^{0}=\varnothing$. Then (S) is satisfied by taking $\Omega=\{\alpha, \beta\}$, where $\alpha<\beta$ in $Z$ (and, necessarily, $\alpha \in \operatorname{Min} Z$ and $\beta \in \operatorname{Max} Z$ ).

Proof. (i) Let $z \in Z$. Given any point $\alpha$ of $Z^{0}$ it is possible to find an orderpreserving map $\theta: Z \rightarrow Z$ which takes $\operatorname{Max} Z$ into $\operatorname{Max} Z$ and $\operatorname{Min} Z$ into $\operatorname{Min} Z$ and which sends $\alpha$ to $z$. 
(ii) Let $z \in \operatorname{Min} Z$ and let $\alpha \in \operatorname{Min} Z$. Then there exists an order-preserving $\operatorname{map} \theta: Z \rightarrow Z$ which takes $\operatorname{Max} Z$ into $\operatorname{Max} Z$ and $\operatorname{Min} Z$ into $\operatorname{Min} Z$ and which sends $\alpha$ to $z$. Similarly, any given point of $\operatorname{Max} Z$ may be sent to any other point of Max $Z$ by a morphism of $\mathcal{D} \mathcal{S}$-spaces.

We next need to identify the piggybacking subalgebras. Again we adopt the notation of Theorem 4.1. In the case of double Stone algebras, or more generally Cornish algebras, there is a unique maximal subalgebra of $(\alpha, \beta)^{-1}(\leq)$ for each $\alpha, \beta \in Z$. This unique subalgebra can be obtained algebraically, by applying Lemma 3.5 of 19], or by using Priestley duality, in the manner described for example in [33, Section 5 .

When $\mathcal{D}=\mathcal{D S}$ and $\mathbf{D}=\mathbf{4}$, we have $Z$ as a 3 -element chain $h(\alpha)<\alpha<g(\alpha)$ and $\Omega=\{\alpha\}$ suffices. The unique maximal subalgebra of $\left\{(x, y) \in D^{2} \mid \alpha(x) \leq \alpha(y)\right\}$ is the partial order

$$
\preccurlyeq:=\{(0,0),(a, a),(a, b),(b, b),(1,1)\},
$$

which as an algebra is isomorphic to $\mathbf{5}$. In this case piggybacking recaptures the natural duality for $\mathcal{D S}$ first obtained by B.A. Davey in [12]. In the terminology of the present paper, $\mathcal{D S}$ is almost endodualisable, with extra relation $\preccurlyeq$.

The structure of the free double Stone algebras is well known. The algebra $\mathbf{F}_{D S}(1)$ is isomorphic to $\mathbf{3} \times \mathbf{2}^{2}$ and the associated $\mathcal{D} \mathcal{S}$-space is just $\{0, a, b, 1\}$ ordered by $\preccurlyeq$. Further, free products of double Stone algebras are given by free bounded distributive lattice products $(30$, p. 327$)$. This means that the $\mathcal{D} \mathcal{S}$ space which is dual to the algebra $\mathbf{F}_{\mathcal{D S}}(k)$ is obtained by taking the $k$-fold power of $(\{0, a, b, 1\} ; \preccurlyeq)$. Certainly, on the finitely generated free algebras, the Priestley duality described in Section 2 coincides with the natural duality between $\operatorname{ISP}(4)$ and $\mathbb{I S}_{c} \mathbb{P}(\mathbf{4})$, the dual category defined as in Section 2 . In fact it can be shown that the two dualities are essentially the same. For a discussion setting this property of double Stone algebras in a wider context see [34, Section 4.

$$
\circ a \circ b \quad{ }^{1}
$$$$
D\left(\mathbf{F}_{\mathcal{D S}}(1)\right)
$$
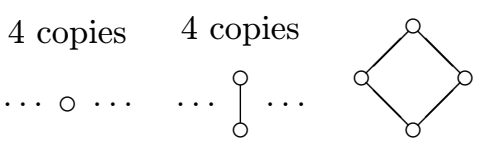

$$
D\left(\mathbf{F}_{\mathcal{D S}}(2)\right)
$$

\section{FiguRE 1.}

The free algebras $F_{\mathcal{D} \mathcal{s}}(1), F_{\mathcal{D} \mathcal{s}}(2)$ and $F_{\mathcal{D} \mathcal{s}}(3)$ play a central role in our discussion of $k$-endoprimality in the next two sections. Their Priestley duals, or equivalently their natural duals, are as depicted in Figures 1 and 2.

Observe that both $F_{\mathcal{D S}}(1)$ and $F_{\mathcal{D S}}(2)$ have $\mathbf{2}$ and $\mathbf{2}^{2}$ as retracts and $F_{\mathcal{D S}}(3)$ has $\mathbf{5} \times \mathbf{2}$ as a retract. Each of these free algebras has $\mathbf{2}$ as a factor, and hence lies in the proper subquasivariety $\mathbb{I S P}(\mathbf{4} \times \mathbf{2})$ of $\mathcal{D} \mathcal{S}$ (see Table 1$)$. Further, $F_{\mathcal{D S}}(1)$ lies in the strictly smaller subquasivariety $\mathbb{I S P}(\mathbf{3} \times \mathbf{2})$. This indicates that we shall be 


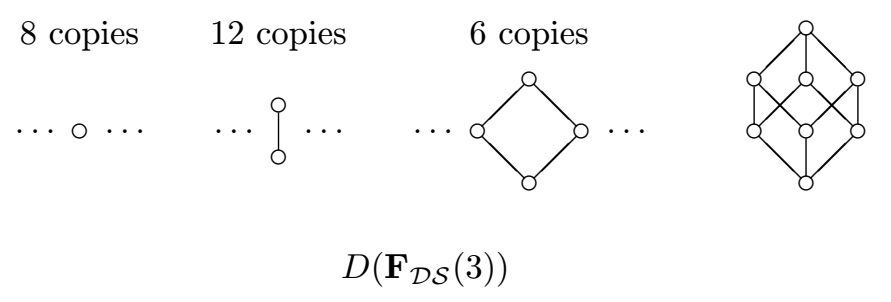

FIGURE 2.

needing dualities for these two subquasivarieties. We can do this by the methods described above. We record the results in the next proposition.

\section{Proposition 4.4.}

(i) $\operatorname{End}(\mathbf{4} \times \mathbf{2}) \cup S$ yields a duality on the subquasivariety $\operatorname{ISP}(\mathbf{4} \times \mathbf{2})$ of $\mathcal{D} \mathcal{S}$, where $S$ contains a single relation, isomorphic as an algebra to $\mathbf{5} \times \mathbf{2}^{2}$.

(ii) $\operatorname{End}(\mathbf{3} \times \mathbf{2}) \cup S$ yields a duality on the subquasivariety $\mathbb{I S P}(\mathbf{3} \times \mathbf{2})$ of $\mathcal{D} \mathcal{S}$, where $S$ contains a single relation, isomorphic as an algebra to $\mathbf{3} \times \mathbf{2}^{2}$.

Proof. Consider (i). We apply Theorem 4.1 with $Z$ as the $\mathcal{D S}$-space which is the disjoint union of one isolated point and a 3-element chain $h(\alpha)<\alpha<g(\alpha)$. By Lemma 4.3 we may take $\Omega=\{\alpha\}$ in Theorem 4.1. The associated subalgebra we must include in the duality is isomorphic to $\preccurlyeq \times \mathbf{2}^{2}$.

For (ii) the appropriate space $Z$ is now the disjoint union of a singleton and a 2 -element chain $\alpha<\beta$, and we can take $\Omega=\{\alpha, \beta\}$. It is easy to check that each of $(\alpha, \alpha)^{-1}(\leq),(\alpha, \beta)^{-1}(\leq)$ and $(\beta, \beta)^{-1}(\leq)$ has a unique maximal $\mathcal{D S}$-subalgebra and that this is isomorphic to $\mathbf{3} \times \mathbf{2}^{2}$ in each case. The unique maximal subalgebra of $(\beta, \alpha)^{-1}(\leq)$ is isomorphic to $\mathbf{2}^{3}$. This is a retract of $\mathbf{3} \times \mathbf{2}^{2}$. Appealing to Lemma 3.2 we get the required result.

We can now rapidly obtain endodualisable double Stone algebras. Recall that any finite double Stone algebra $\mathbf{L}$ is expressible as a product $\mathbf{J} \times \mathbf{2}^{\ell}$, where $\mathbf{J}$ does not have $\mathbf{2}$ as a factor (equivalently, $\mathbf{J}$ is trivial or has a non-empty core) and $\ell \geq 0$.

Theorem 4.5. Let $\mathbf{L}$ be a finite non-trivial double Stone algebra and express $\mathbf{L}$ as $\mathbf{J} \times \mathbf{2}^{\ell}$ where $\mathbf{J}$ does not have $\mathbf{2}$ as a factor and $\ell \geq 0$.

Then $\mathbf{L}$ is endodualisable when $\mathbf{L}$ takes one of the forms described below.

(a) $\mathbf{L}$ has non-empty core and $\mathbf{L}$ satisfies the following equivalent conditions:

(1) $\mathbf{L}$ has $\mathbf{5}$ as a retract,

(2) $K(\mathbf{L})$ is a non-Boolean lattice;

(b) $\mathbf{L}$ is proper, $\mathbf{J}$ has $\mathbf{5}$ as a retract and $\ell \geq 2$;

(c) $\mathbf{L}$ is not proper and takes the form $\mathbf{3}^{m} \times \mathbf{2}^{\ell}$ where $m \geq 1$ and $\ell \geq 2$;

(d) L is Boolean.

Proof. Observe that the cases delineated in the statement concern algebras in the following classes:

(a) $\mathbb{I S P}(\mathbf{4}) \backslash(\mathbb{I S P}(\mathbf{4} \times \mathbf{2}) \cup \operatorname{ISP}(\mathbf{3}))$,

(b) $\operatorname{ISP}(\mathbf{4} \times \mathbf{2}) \backslash \mathbb{I S P}(\mathbf{3})$, 
(c) $\mathbb{I S P}(\mathbf{3} \times \mathbf{2}) \backslash \mathbb{I S P}(\mathbf{2})$,

(d) $\operatorname{ISP}(\mathbf{2})$.

In case (a), $\mathbf{L}$ has both $\mathbf{4}$ and $\mathbf{5}$ as retracts (see Table 1) and we can apply Theorem 3.4 with $\mathbf{D}=\mathbf{4}$ and $\mathrm{s}$ as $\preccurlyeq$.

In case (b), we need the duality for $\mathbb{I S P}(\mathbf{4} \times \mathbf{2})$ given in Theorem 4.4(i). In Theorem 3.4 we take $\mathbf{D}=\mathbf{4} \times \mathbf{2}$ and $\mathbf{s}=\preccurlyeq \times \mathbf{2}^{2}$.

In case (c), $\mathbf{L}$ has as retracts both $\mathbf{D}:=\mathbf{3} \times \mathbf{2}$ and $\mathbf{s}:=\mathbf{3} \times \mathbf{2}^{2}$. Thus $\mathbf{L}$ is endodualisable, by Theorem 4.4(b) and Theorem 3.4.

There is nothing to prove in case (d).

\section{Non-endodualisable $\mathcal{D} \mathcal{S}$-algebras and the failure of $k$-endoprimality}

Assume we are given some finite algebra $\mathbf{M}$ which we know to be almost endodualisable with extra relation $s$ and that we wish to show that $\mathbf{M}$ is not endodualisable and to identify a value of $k$ such that $\mathbf{M}$ is not $k$-endoprimal. We may seek to exhibit, for some $k$, a function $f: M^{k} \rightarrow M$ which preserves End $\mathbf{M}$ and is not a term function. By Proposition 3.1 this is equivalent to showing that $\mathbf{F}_{\mathbb{I S P}(\mathbf{M})}(k)$ serves as a test algebra witnessing non-endodualisability. Further, we can confirm that $f$ cannot be a term function by checking that $f$ fails to preserve the relation $s$, which we extend co-ordinatewise to powers of $M$; in fact it is enough to show that any chosen algebraic relation is not preserved. Alternatively, we may endeavour to show directly that $\mathbf{s}$ serves as a test algebra witnessing non-endodualisability. In taking this route we would seek to choose our test algebra to be a retract of a $k$-generated free algebra, so that Proposition 3.3 applies. Taking account of our comments concerning free double Stone algebras in the preceding section and the information in Table 1 we have the following lemma.

Lemma 5.1. Assume that $\mathbf{L}$ is a finite double Stone algebra which is not endodualisable and that End $\mathbf{L}$ does not yield a duality on a test algebra $\mathbf{s}$.

(i) If $\mathbf{s} \cong \mathbf{2}$ or $\mathbf{s} \cong \mathbf{2} \times \mathbf{2}$ then $\mathbf{L}$ is not 1-endoprimal.

(ii) If $\mathbf{s} \cong \mathbf{5} \times \mathbf{2}$ then $\mathbf{L}$ is not 3-endoprimal.

Let $\mathbf{L}$ be a finite non-trivial and non-Boolean double Stone algebra which is not shown by Theorem 4.5 to be endodualisable and assume that $\mathbf{L}$ is expressed as $\mathbf{J} \times \mathbf{2}^{\ell}$ where $\mathbf{J}$ does not have $\mathbf{2}$ as a factor. The following cases arise:

(A) $\mathbf{L}$ is a Post algebra of order 3 (that is, $\mathbf{L}$ is not proper and $\ell=0$ );

(B) $\mathbf{L}$ has a single factor $\mathbf{2}$ (that is, $\ell=1$ );

(C) $\mathbf{L}$ is proper, $K(\mathbf{L}) \neq \varnothing$ (that is, $\ell=0$ ), and $\mathbf{J}$ does not have $\mathbf{5}$ as a retract;

(D) $\mathbf{L}$ is proper, $\mathbf{J}$ does not have $\mathbf{5}$ as a retract and $\ell \geq 2$.

We shall show that $\mathbf{L}$ is not endodualisable in each of cases (A)-(D), treating these in turn.

Proposition 5.2. Let $\mathbf{L}$ be a finite Post algebra of order 3 . Then

(i) $\mathbf{L}$ is not endodualisable, with $\mathbf{2}$ serving as a test algebra;

(ii) $\mathbf{L}$ is not 1-endoprimal. 
Proof. The algebra $\mathbf{L}$ is of the form $\mathbf{3}^{n}$, for some $n \geq 1$. It has a one-element core, $K(\mathbf{L})=\{c\}$, say. We take the unary relation $s=\{0,1\} \cong \mathbf{2} \leqslant \mathbf{L}$. The dual $D(\mathbf{s}):=\operatorname{hom}(\mathbf{s}, \mathbf{L})$ contains a single element, $\rho$ say. Define $\varphi: D(\mathbf{s}) \rightarrow \mathbf{L}$ by $\varphi(\rho)=c$. It is easy to see that $\varphi$ preserves every endoaction of End $\mathbf{L}$ on $D(\mathbf{s})$. On the other hand, $\rho \in s_{D(\mathbf{s})}$, but $\varphi(\rho) \notin s$, so $\varphi$ does not preserve $s$.

(We remark that we could alternatively have proceeded as follows. We can first show, easily, that $\mathbf{3}$ is not 1-endoprimal, using the constant unary function mapping $\mathbf{3}$ onto its one-element core. Theorem 3.6 then implies that any finite power of $\mathbf{3}$ is also not 1-endoprimal.)

Proposition 5.3. Let $\mathbf{L}=\mathbf{J} \times \mathbf{2}$ be a finite non-Boolean double Stone algebra with exactly one factor $\mathbf{2}$. Then

(i) $\mathbf{L}$ is not endodualisable, with $\mathbf{2}^{2}$ serving as a test algebra;

(ii) $\mathbf{L}$ is not 1-endoprimal.

Proof. We prove (i). Part (ii) then follows immediately from Lemma 5.1. We first note that $\mathbf{2}^{2}$ may be regarded as a subalgebra of $\mathbf{J} \times \mathbf{2}$, and so certainly belongs to $\mathbb{I S P}(\mathbf{L})$. We shall show that End $\mathbf{L}$ fails to yield a duality on the algebra $\mathbf{2}^{2}$.

Suppose that $\mathbf{J}$ is a direct product of $k$ indecomposable factors $\mathbf{L}_{1}, \ldots, \mathbf{L}_{k}$ different from $\mathbf{2}$, and put $\mathbf{L}_{k+1}=\mathbf{2}$.

The algebra $\mathbf{J} \times \mathbf{2}$ has a maximal Boolean subalgebra $\mathbf{B}$ isomorphic to $\mathbf{2}^{k+1}$. We appeal once again to Lemma 2.1 to describe the endomorphisms of $\mathbf{L}$. We see that every endomorphism $e$ on $\mathbf{J} \times \mathbf{2}$ takes the form

$$
e\left(\left(a_{1}, \ldots, a_{k}, a_{k+1}\right)\right)=\left(e_{1}\left(a_{\epsilon(1)}\right), \ldots, e_{k}\left(a_{\epsilon(k)}\right), a_{k+1}\right)\left(\left(a_{1}, \ldots, a_{k}\right) \in \mathbf{J}, a_{k+1} \in \mathbf{2}\right),
$$

where $\epsilon:\{1, \ldots, k\} \rightarrow\{1, \ldots, k+1\}$ and $e_{i}$ is a homomorphism from $\mathbf{L}_{\epsilon(i)}$ to $\mathbf{L}_{i}$. The fixing of the last co-ordinate occurs because $\operatorname{hom}\left(\mathbf{L}_{i}, \mathbf{2}\right)=\varnothing$ for $i \leq k$ and $\operatorname{hom}(\mathbf{2}, \mathbf{2})=\mathrm{id}_{\mathbf{2}}$. Note further that $e$ must map $\mathbf{B}$ into itself. Again invoking Lemma 2.1 we see that the elements of $D\left(\mathbf{2}^{2}\right)=\operatorname{hom}\left(\mathbf{2}^{2}, \mathbf{J} \times \mathbf{2}\right)$ are in one-to-one correspondence with the elements of $\mathbf{B}$. In fact each is a Boolean homomorphism from the free Boolean algebra $\mathbf{F}_{\mathcal{B}}(1)$ into $\mathbf{B} \leqslant \mathbf{J} \times \mathbf{2}$, determined by the image $\left(b_{1}, \ldots, b_{k}, b_{k+1}\right) \in B$ of the free generator $(1,0)$, and so may be written as $v\left[b_{1}, \ldots, b_{k}, b_{k+1}\right]$, where

$$
v\left[b_{1}, \ldots, b_{k}, b_{k+1}\right](1,0)=\left(b_{1}, \ldots, b_{k}, b_{k+1}\right) .
$$

Alternatively, this description can easily be obtained by Priestley duality. Now observe that, for an endomorphism $e$ as given above,

$$
e \circ v\left[b_{1}, \ldots, b_{k}, b_{k+1}\right]=v\left[b_{\epsilon(1)}, \ldots, b_{\epsilon(k)}, b_{k+1}\right] .
$$

Note in particular that the right-hand side depends only on the map $\epsilon$ and not on the maps $e_{i}$, and that the final co-ordinate, $b_{k+1}$, is left unchanged under the endoaction. Further, we see that, given $\left(b_{1}, \ldots, b_{k}, b_{k+1}\right) \in \mathbf{2}^{k+1}$, we can find an endomorphism $e$ such that

$$
v\left[b_{1}, \ldots, b_{k}, b_{k+1}\right]= \begin{cases}e \circ v[0, \ldots, 0,1] & \text { if } b_{k+1}=1, \\ e \circ v[1, \ldots, 1,0] & \text { if } b_{k+1}=0 .\end{cases}
$$


We can now define a map $\varphi: D\left(\mathbf{2}^{2}\right) \rightarrow \mathbf{B}$ which preserves End $\mathbf{L}$ in the following way. We first choose $\varphi(v[0, \ldots, 0,1])$ and $\varphi(v[1, \ldots, 1,0])$ arbitrarily from the fourelement set

$$
\left\{\left(\mathbf{0}_{\mathbf{J}}, 0\right),\left(\mathbf{1}_{\mathbf{J}}, 0\right),\left(\mathbf{0}_{\mathbf{J}}, 1\right),\left(\mathbf{1}_{\mathbf{J}}, 1\right)\right\},
$$

where $\mathbf{0}_{\mathbf{J}}$ and $\mathbf{1}_{\mathbf{J}}$ are the zero and identity of $\mathbf{J}$. We can then extend $\varphi$ to $D\left(\mathbf{2}^{2}\right)$ by requiring that $\varphi$ preserve the endoaction; the facts noted above ensure that we do indeed get a well-defined map on the whole of $D\left(\mathbf{2}^{2}\right)$. Therefore the second dual of $\mathbf{2}^{2}$ contains at least sixteen elements. This proves our claim that End $\mathbf{L}$ does not yield a duality on $\mathbf{2}^{2}$.

For case $(C)$ we show that the algebra $\mathbf{L}$ is the retract of a power of a finite indecomposable algebra which is not 3-endoprimal. Thanks to Lemma 2.1 we are then able to employ Theorem 3.6 .

Lemma 5.4. Let $\mathbf{L}$ be the double Stone algebra $\mathbf{A}_{n}(n \geq 1)$. Then $\mathbf{L}$ is not 3-endoprimal. In particular $\mathbf{4}=\mathbf{A}_{1}$ is not 3-endoprimal.

Proof. An endomorphism of any double Stone algebra maps the core into the core. Here we have the special property that $\mathbf{L} \backslash K(\mathbf{L})=\{0,1\}$ and this implies that $\mathbf{L} \backslash K(\mathbf{L})$ is fixed by End $\mathbf{L}$.

The core of $\mathbf{L}$ is a non-empty interval $[a, b]$ with $a<b$. We take the ternary function $f: \mathbf{L}^{3} \rightarrow \mathbf{L}$ by defining $f(x, y, z)$ to be the relative complement of $y$ in the interval $[x \wedge y \wedge z, x \vee y \vee z]$ if $x, y, z \in K(\mathbf{L})$ and $f(x, y, z)=x$ otherwise. (The motivation for the way $f$ is defined to act on the Boolean core comes from [31, where the relative complement is used to prove that a Boolean lattice is not 3-endoprimal; see also [15]). Because any element of End $\mathbf{L}$ maps $K(\mathbf{L})$ into itself and $\mathbf{L} \backslash K(\mathbf{L})$ into itself, it is easy to see that $f$ preserves End $\mathbf{L}$. Let $f^{\prime}$ denote the restriction of $f$ to the core $[a, b]$. Observe that $f^{\prime}$ does not preserve the lattice order, $\leq$, on $[a, b]$ because $f^{\prime}(a, a, b)=b>a=f^{\prime}(a, b, b)$. But this could not happen if $f$ were a term function. If it were, then $f^{\prime}$ would be a lattice term on $[a, b]$ (because, on the core, the terms $x^{*}$ and $x^{+}$only take values 0 or 1 ). Alternatively, we may reach the same conclusion by observing that $f$ fails to preserve $\preccurlyeq$, interpreted as the extra relation $\{(0,0),(a, a),(a, b),(b, b),(1,1)\}$ in the natural duality for $\mathbb{I S P}(\mathbf{L})=\mathbb{I S P}(\mathbf{4})$ derived using Theorem 4.1.

Proposition 5.5. Let $\mathbf{L}$ be a finite proper double Stone algebra with a non-empty core $K(\mathbf{L})=[a, b](a<b)$ which is a Boolean lattice. Then $\mathbf{L}$ is not 3-endoprimal (and hence not endodualisable).

Proof. The assumptions on $\mathbf{L}$ imply that we may express $\mathbf{L}$ as a finite product $\mathbf{A}_{j_{1}} \times \cdots \times \mathbf{A}_{j_{m}}$, where $j_{1} \geq \cdots \geq j_{m} \geq 0$ and $j_{1}>0$. Then $\mathbf{L}$ is a retract of $\mathbf{M}:=\mathbf{P}^{m}$, where $\mathbf{P}:=\mathbf{A}_{j_{1}}$. Further, $\operatorname{ISP}(\mathbf{L})=\mathbb{I S P}(\mathbf{M})(=\mathbb{I S P}(\mathbf{4}))$. Therefore, by Theorem 3.5, it will suffice to show that $\mathbf{M}$ is not 3-endoprimal. We have already proved in Lemma 5.4 that $\mathbf{P}$ is not 3-endoprimal. We apply Theorem 3.6 to complete the proof.

Finally we need to consider algebras which have $\mathbf{2}^{\ell}$ as a factor, where $\ell \geq 2$ (case $(\mathrm{D}))$. We do not use the fact that $\ell>1$ in the proof below, but in the 
case $\ell=1$ we already have a stronger conclusion than that in Proposition 5.6. Our method is similar to that for case (C); we exploit the fact that there is no homomorphism from a non-trivial finite double Stone algebra with a non-empty core to the algebra 2.

Proposition 5.6. Let $\mathbf{L}=\mathbf{J} \times \mathbf{2}^{\ell}$, where $\mathbf{J} \in \mathbb{I S P}(\mathbf{4}) \backslash \mathbb{I S P}(\mathbf{3})$ is a finite double Stone algebra with a non-trivial Boolean core and $\ell \geq 2$. Then $\mathbf{L}$ is not 3 -endoprimal (and so not endodualisable).

Proof. The algebra $\mathbf{J}$ is a retract of a power $\mathbf{M}^{p}$ of an indecomposable algebra $\mathbf{M}$ which is of the form $\mathbf{A}_{n}$ for some $n \geq 1$. The algebra $\mathbf{L}$ is then a retract of $\mathbf{M}^{p} \times \mathbf{2}^{\ell}$ (where $p \geq 1$ and $\ell \geq 2$ ), and it will suffice to show that algebras $\mathbf{L}$ of this special type are not 3 -endoprimal.

The algebra $\mathbf{M}$ is shown by the ternary function $f: M^{3} \rightarrow M$, as given in the proof of Lemma 5.4, to fail to be 3-endoprimal. We shall need the facts that $f(x, y, z)=x$ whenever $x, y, z \in\{0,1\}$ and that End $\mathbf{M}$ fixes the subalgebra $\{0,1\}$. We shall use $f$ to define a ternary function $g: L^{3} \rightarrow L$ which witnesses the failure of 3 -endoprimality. We denote elements of $\mathbf{L}$ by vectors $\mathbf{a}=\left(a_{1}, \ldots, a_{p}, a_{p+1}, \ldots, a_{p+\ell}\right)$ where $a_{i} \in M$ for $i \leq p$ and $a_{i} \in\{0,1\}$ for $i>p$. We define

$$
g(\mathbf{x}, \mathbf{y}, \mathbf{z})=\left(f\left(x_{1}, y_{1}, z_{1}\right), \ldots, f\left(x_{p}, y_{p}, z_{p}\right), x_{p+1}, \ldots, x_{p+\ell}\right) .
$$

As usual we appeal to Lemma 2.1 to describe the endomorphisms of $\mathbf{L}$. In this case an endomorphism $e$ of $\mathbf{L}$ takes the form

$$
e\left(\left(a_{1}, \ldots, a_{p+\ell}\right)\right)=\left(e_{1}\left(a_{\epsilon(1)}\right), \ldots, e_{p}\left(a_{\epsilon(p)}\right), e_{p+1}\left(a_{\epsilon(p+1)}\right), \ldots, e_{p+\ell}\left(a_{\epsilon(p+\ell)}\right)\right),
$$

where

(i) $\epsilon:\{1, \ldots, p+\ell\} \rightarrow\{1, \ldots, p+\ell\}$ is a map such that, whenever $i>p, \epsilon(i)>p$ and $e_{i}$ is the identity map on $\mathbf{2}$,

(ii) $e_{i} \in$ End $\mathbf{M}$ if $i \leq p$ and $\epsilon(i) \leq p$, and

(iii) $e_{i}$ is the (unique) embedding of $\mathbf{2}$ into $\mathbf{M}$ if $i \leq p$ and $\epsilon(i)>p$.

Here we have used the fact hom $(\mathbf{M}, \mathbf{2})=\varnothing$. For any $i$ we have, using the facts that $f$ preserves End $\mathbf{M}$ and $e_{i}$ fixes 0 and 1 ,

$$
\begin{aligned}
\pi_{i}(e(g(\mathbf{x}, \mathbf{y}, \mathbf{z}))) & = \begin{cases}e_{i}\left(f\left(x_{\epsilon(i)}, y_{\epsilon(i)}, z_{\epsilon(i)}\right)\right) & \text { if } \epsilon(i) \leq p \\
e_{i}\left(x_{\epsilon(i)}\right) & \text { otherwise }\end{cases} \\
& = \begin{cases}f\left(e_{i}\left(x_{\epsilon(i)}\right), e_{i}\left(y_{\epsilon(i)}\right), e_{i}\left(z_{\epsilon(i)}\right)\right) & \text { if } \epsilon(i) \leq p \\
x_{\epsilon(i)} & \text { otherwise }\end{cases} \\
& =\pi_{i}(g(e(\mathbf{x}), e(\mathbf{y}), e(\mathbf{z}))) .
\end{aligned}
$$

For the final equality here we need the fact that, if $i \leq p$ and $\epsilon(i)>p$, then $f\left(\left(e_{i}\left(x_{\epsilon(i)}\right), e_{i}\left(y_{\epsilon(i)}\right), e_{i}\left(z_{\epsilon(i)}\right)\right)\right)=e_{i}\left(x_{\epsilon(i)}\right)$.

The function $f$ fails to preserve the binary relation $\preccurlyeq$, which as a subalgebra of $\mathbf{M}^{2}$ is isomorphic to $\mathbf{5}$. We can construct a subalgebra $\mathbf{s} \leqslant \mathbf{L}^{2}$ isomorphic to $\mathbf{5} \times \mathbf{2}$ consisting of pairs $(\mathbf{x}, \mathbf{y})$ such that $x_{i} \preccurlyeq y_{i}$ for $i \leq p$ and $x_{p+1}=\cdots=$ $x_{p+\ell}=y_{p+1}=\ldots y_{p+\ell}$. Then $g$ fails to preserve $s$. Consequently, $\mathbf{L}$ cannot be 3 -endoprimal. 
We remark that we could have obtained the preceding result by appealing to Lemma 5.1, using $\mathbf{5} \times \mathbf{2}$ as our test algebra. To carry this through we have to use Lemma 2.1 to describe the elements of $\operatorname{hom}\left(\mathbf{5} \times \mathbf{2}, \mathbf{J} \times \mathbf{2}^{\ell}\right)$ and to define an endomorphism-preserving function from this hom-set to $\mathbf{J} \times \mathbf{2}^{\ell}$ which does not preserve the test relation $s$, where $\mathbf{s} \cong \mathbf{5} \times \mathbf{2}$. This is feasible, but notationally complicated.

\section{Conclusions}

We identified various endodualisable finite double Stone algebras in Section 4 and have shown in Section 5 that there are no other such algebras. Here we bring our results together and add some further remarks.

Theorem 6.1. Let $\mathbf{L}=\left(L ; \vee, \wedge,{ }^{\star},{ }^{+}, 0,1\right)$ be a finite proper double Stone algebra with a non-empty core $K(\mathbf{L})=[a, b](a<b)$. Then the following are equivalent:

(1) $\mathbf{L}$ is endodualisable;

(2) $\mathbf{L}$ is endoprimal;

(3) $\mathbf{L}$ is 3-endoprimal;

(4) $\mathbf{5}$ is a retract of $\mathbf{L}$;

(5) the core $K(\mathbf{L})$ is a non-Boolean lattice.

Proof. Note that $(1) \Rightarrow(2)$ and $(2) \Rightarrow(3)$ are true always, (3) $\Rightarrow(5)$ was proved in Proposition 5.5 and $(4) \Leftrightarrow(5)$ was proved in Lemma 2.3. To complete the proof we appeal to Theorem 4.5 .

We observe that this theorem fits into the framework for endoprimality characterisations from [17. Make the following assumptions on an algebra D:

(a) $\mathbf{D}$ is almost endodualisable via $\underset{\sim}{\mathbf{D}}=(D ; \operatorname{End} \mathbf{D}, s, \tau)$;

(b) $\mathbf{E}$ is a finite algebra in the quasivariety $\mathcal{D}=\mathbb{I S P}(\mathbf{D})$ isomorphic to $\mathbf{s}$ which has $\mathbf{D}$ as a retract;

(c) Steps (1) and (2) below can be carried out in $\mathcal{D}$ with some $k \geqslant 1$ :

(1) find an internal property, call it $(\varepsilon)$, such that, for all $\mathbf{M} \in \mathcal{D}$, the algebra $\mathbf{E}$ is a retract of $\mathbf{M}$ if and only if $\mathbf{M}$ satisfies $(\varepsilon)$, and

(2) prove that, for some $k \in \mathbb{N}$, if $\mathbf{M} \in \mathcal{D}$ has $\mathbf{D}$ as a retract and does not satisfy $(\varepsilon)$, then $\mathbf{M}$ is not $k$-endoprimal.

Then the following are equivalent:

(i) $\mathbf{M}$ is endoprimal;

(ii) $\mathbf{M}$ is $k$-endoprimal;

(iii) $\mathbf{E}$ is a retract of $\mathbf{M}$;

(iv) $\mathbf{M}$ satisfies $(\varepsilon)$.

Moreover, if $\mathbf{M}$ is finite, then these conditions are also equivalent to

(v) $\mathbf{M}$ is endodualisable.

In our Theorem 6.1, $\mathbf{E}=\mathbf{5}$ and the internal property $(\varepsilon)$ : 'the core $K(\mathbf{L})$ is a non-Boolean lattice'. Observe that $\mathbf{L} \in \mathcal{D S}$ has $\mathbf{4}$ as a retract if and only if its core has at least two elements, so that $K(\mathbf{L})=[a, b]$ where $a<b$. 
For proper double Stone algebras with empty core we have the following theorem.

Theorem 6.2. Let $\mathbf{L}=\left(L ; \vee, \wedge,{ }^{\star},{ }^{+}, 0,1\right)$ be a finite proper double Stone algebra with an empty core. Then the following are equivalent:

(i) $\mathbf{L}$ is endodualisable;

(ii) $\mathbf{L}$ is endoprimal;

(iii) $\mathbf{L}$ is 3-endoprimal;

(iv) $\mathbf{5} \times \mathbf{2}^{2}$ is a retract of $\mathbf{L}$.

For algebras in $\operatorname{ISP}(3)$ we have, likewise, the following result.

Theorem 6.3. Let $\mathbf{L}$ belong to the variety $\mathcal{R}=\mathbb{I S P}(\mathbf{3})$ of regular double Stone algebras and assume that $\mathbf{L}$ is not Boolean. Then the following are equivalent:

(1) $\mathbf{L}$ is endodualisable;

(2) $\mathbf{L}$ is endoprimal;

(3) $\mathbf{L}$ is 1-endoprimal;

(4) $\mathbf{3} \times \mathbf{2}^{2}$ is a retract of $\mathbf{L}$.

We record explicitly the following theorem, which is a corollary of our preceding results.

Theorem 6.4. A finite double Stone algebra is endoprimal if and only if it is endodualisable.

\section{REFERENCES}

1. M.E. Adams, M. Atallah and R. Beazer Congruence coherent distributive double p-algebras, Proc. Edinburgh Math. Soc. 39 (1996), 71-80.

2. R. Beazer A note on injective double Stone algebras, Algebra Universalis 5 (1975), 239-241.

3. R. Beazer The determination congruence on double p-algebras, Algebra Universalis 6 (1976), $121-129$.

4. R. Beazer Some remarks on distributive double p-algebras, Algebra Universalis 8 (1978), 9-14.

5. R. Beazer Congruence pairs of distributive double p-algebras with nonempty core, Houston J. Math. 6 (1980), 443-454.

6. R. Beazer Subdirectly irreducibles for various pseudocomplemented algebras, Algebra Universalis 10 (1980), 225-231.

7. R. Beazer Affine complete double Stone algebras with bounded core, Algebra Universalis 16 (1983), 237-244.

8. R. Beazer Distributive p-algebras and double p-algebras having $n$-permutable congruences, Proc. Edinburgh. Math. Soc. 35 (1992), 301-307.

9. D.M. Clark and B.A. Davey, Natural Dualities for the Working Algebraist, Cambridge University Press, Cambridge, 1998.

10. W.H. Cornish Antimorphic Action. Categories of Algebraic Structures with Involutions or Anti-endomorphisms, R \& E Research and Exposition in Mathematics 12, Heldermann Verlag, Berlin, 1986.

11. B.A. Davey Dualities for equational classes of Brouwerian algebras and Heyting algebras, Trans. Amer. Math. Soc. 221 (1976), 119-146.

12. B.A. Davey Dualities for Stone algebras, double Stone algebras, and relative Stone algebras, Colloq. Math. 46 (1982), 1-14.

13. B.A. Davey Duality theory on ten dollars a day, Algebras and Orders (I.G. Rosenberg and G. Sabidussi, eds), NATO Advanced Study Institute Series, Series C, Vol. 389, Kluwer Academic Publishers, 1993, 71-111. 
14. B.A. Davey, Dualisability in general and endodualisability in particular, Logic and algebra (Pontignano, 1994) (A. Ursini and P. Aglianò, eds), Lecture Notes in Pure and Appl. Math., 180, Dekker, New York, 1996, 437-455.

15. B.A. Davey, M. Haviar and H.A. Priestley Endoprimal distributive lattices are endodualisable, Algebra Universalis 34 (1995), 444-453.

16. B.A. Davey, M. Haviar and H.A. Priestley The syntax and semantics of entailment in duality theory, J. Symbolic Logic 60 (1995), 1087-1114.

17. B.A. Davey and J.G. Pitkethly Endoprimal algebras, Algebra Universalis 38 (1997), 266-288.

18. B.A. Davey and H.A. Priestley, Introduction to Lattices and Order, Cambridge University Press, Cambridge, 1990 (second edition 2002).

19. B.A. Davey and H.A. Priestley Generalised piggyback dualities and applications to Ockham algebras, Houston J. Math. 13 (1987), 151-197.

20. B.A. Davey and H.A. Priestley Optimal natural dualities, Trans. Amer. Math. Soc. 338 (1993), 655-677.

21. B.A. Davey and H.A. Priestley Optimal natural dualities II: general theory, Trans. Amer. Math. Soc. 348 (1996), 3673-3711.

22. B.A. Davey and H.A. Priestley Optimal natural dualities for varieties of Heyting algebras, Studia Logica 56 (1996), 67-96.

23. I. Düntsch On free or projective regular double Stone algebras, Houston J. Math. 9 (1983), 455-463.

24. I. Düntsch Finite projective double Stone algebras, Proc. Roy. Soc. Edinburgh (Series A) 96 (1984), 65-67.

25. G. Grätzer, General Lattice Theory (2nd edition), Appendix B: Distributive Lattices, by B. A. Davey and H.A. Priestley, Birkhäuser Verlag, Basel, 1998.

26. M. Haviar and H.A. Priestley A criterion for a finite endoprimal algebra to be endodualisable, Algebra Universalis 42 (1999), 183-193.

27. T. Katriňák The structure of distributive double p-algebras. Regularity and congruences, Algebra Universalis 3 (1973), 238-246.

28. T. Katriňák Injective double Stone algebras, Algebra Universalis 4 (1974), 259-267.

29. T. Katriňák Construction of regular double p-algebras, Bull. Soc. Roy. Liege 43 (1974), 283 290.

30. T. Katriňák The free $\{0,1\}$-distributive product of distributive p-algebras, Bull. Soc. Roy. Liege 47 (1978), 323-328.

31. L. Márki and R. Pöschel Endoprimal distributive lattices, Algebra Universalis 30 (1993), $272-274$.

32. H.A. Priestley Stone lattices: a topological approach, Fund. Math. 84 (1974), 127-143.

33. H.A. Priestley Natural dualities, Lattice Theory and its Applications - A Volume in Honor of Garrett Birkhoff's 80th Birthday (K.A. Baker and R. Wille, eds), Heldermann Verlag, Berlin, 1995, 185-209.

34. H.A. Priestley Varieties of distributive lattices with unary operations I, J. Austral. Math. Soc. (Series A) 63, (1997), 165-207.

35. M. Saramago, A study of natural dualities including an analysis of the structure of failsets, Ph.D. thesis, University of Lisbon, 1999.

Mathematics Department, Matej Bel University, 97401 Banská Bystrica, Slovakia

E-mail address: haviar@pdf.umb.sk

Mathematical Institute, University of Oxford, 24/29 St. Giles, Oxford OX1 3LB, UNITED KINGDOM

E-mail address: hap@maths.ox.ac.uk 